\title{
Advancing Psychology as a Bio-behavioral Science
}

\author{
John E. Carr
}

Published online: 7 February 2008

(C) The Author(s) 2008

\begin{abstract}
Concerns for the integrity of psychology as an independent discipline have caused some psychologists to object to introducing any knowledge from the biological sciences into the training of psychologists. However, calls for the greater incorporation of the behavioral sciences in medical education, increased attention to research on the mechanisms of bio-behavioral interaction, and initiatives in translational medical research and clinical care, have prompted increased interest in interdisciplinary research, health care, and teaching. These changes, in turn, are resulting in a reconceptualization of the structure of academic medicine with increasing emphasis upon multidisciplinary knowledge and interdisciplinary collaboration, and less emphasis upon disciplinary insularity and competitiveness. If clinical health psychology is to play a role in this evolving concept of academic health care, it must adequately prepare its trainees to function in interdisciplinary academic health care settings. This will require not only expertise in the role of behavioral factors relevant to medical disorders, but also some basic familiarity with the biological processes to which those behavioral factors relate. With the evolution of its fund of knowledge, clinical health psychology has the potential to utilize its science to discover, describe, interpret,
\end{abstract}

Presented, in part, to the Association of Psychologists in Academic Health Centers, Minneapolis, MN, May 2007.

\section{J. E. Carr $(\square)$}

Departments of Psychiatry \& Behavioral Sciences, and Psychology, University of Washington, 4225 Roosevelt Way, NE, Seattle, WA 98105, USA

e-mail: jcarr@u.washington.edu teach and clinically apply knowledge of the mechanisms of interaction between biological functions and behavioral, learning, cognitive, socio-cultural and environmental processes. By failing to seize this initiative, clinical health psychology risks becoming irrelevant to the evolving model of medical research, education and health care.

Keywords Clinical health psychology ·

Bio-behavioral science . Academic health care settings

\section{Introduction}

The establishment of postdoctoral training courses in pharmacology for licensed clinical psychologists is seen by some as an inevitable precursor to seeking limited prescription privileges. Since anything that contributes to the medicalization of psychology, is presumed by some to "...ring the death knell for psychology as a distinctive and independent professional practice" (Sonnanburg, 2007), the merit and value of such courses is too often judged solely on that basis. However, it can be argued that whether psychologists should or should not prescribe is irrelevant to the more important issue of the evolution of clinical health psychology as a science, and the future education of psychology trainees. While there are rationales for monitoring the integrity of psychology as a distinct and independent discipline, to assert that knowledge from pharmacology, neurology, psychiatry, physiology, and biochemistry "is NOT psychology", or has no place in psychology, impedes its evolution as a science and puts its relevance and viability as a health care profession at significant risk, especially in light of recent developments in medical science, practice, and education. 


\section{The Institute of Medicine Report on Behavioral Sciences in Medical Education}

For more than 30 years researchers have documented the importance of behavioral factors in disease and health care. Over that same period of time medical educators have persistently called for the greater inclusion of the behavioral sciences in medical education, only to have those recommendations repeatedly ignored. In 2004, the Institute of Medicine (IOM) published a report identifying barriers to the integration of the behavioral sciences and specifying priority behavioral science topics to be incorporated in medical school curricula (Cuff \& Vanselow, 2004).

The primary barrier was identified as administrators of academic medical centers who seemed to lack familiarity with or exposure to the behavioral sciences and their research literature. It is reasonable to assume that administrators are not individuals of limited intelligence or duplicitous motives with regard to the behavioral sciences. How then do we account for their lack of familiarity and exposure? The principle problem lies in the historical legacy of the Flexner Commission (Flexner, 1910) which recommended the establishment of scientifically based medicine, but on exclusively biological foundations. The effect was to foster the development of medical school structures involving biological discipline-specific departments (e.g. anatomy, physiology, etc). Behavioral science departments did not exist in medical schools at the time and therefore played little or no role in the administration of academic medicine until late in the 20th century. Decisions regarding the allocation of resources, teaching time, curriculum content and faculty positions were, and continue to be, made by the (biological) discipline specific departments, their heads and the Deans who are promoted from those departments. The legacy of Flexnerian biomedicine gave rise to the concept of the "hidden curriculum", a concept coined by sociologist Philip Jackson in 1968, describing the subtle socialization process by which doctors "learn" about the primacy of the physician in the health care system, biological fundamentalism, and the limited relevance of the behavioral sciences to medical practice (see Carr, Emory, Errichetti, Bennett Johnson, \& Reyes, 2007, for a more detailed discussion of the implications of the IOM report for psychology).

Thus, not only do medical school administrators need to be enlightened and trained, but the entire structure of health care and medical education requires significant restructuring. Surprisingly, and as improbable as this seems, current developments are taking place that have profound implications for the future conceptualization of medicine and the structure of medical education and, by extension, graduate education in psychology.

\section{Bio-behavioral Interaction-The Missing Link}

The IOM recommended and the National Institutes of Health (NIH) have established career and program development grants in the behavioral sciences. In its report the IOM also recommended greater inclusion of the following six topic domains from the behavioral sciences in medical education:

(1) Mind-body interactions, the mechanisms of interaction among biological, behavioral, cognitive, sociocultural and environmental contributions to disease and health;

(2) Patient behavior focusing on the principles of behavior change that play a role in disease and disease prevention;

(3) Physician role and behavior and the attitudes, beliefs and behavior that influence physician efficacy;

(4) Physician-patient interaction and communication;

(5) Social and cultural issues that contribute to health care, cultural competence and social inequalities in health care;

(6) Health policy and economics, the health care system, financial incentives and other policy and economic influences on health care delivery.

The topics in domains 2-6 are already well represented in the curricula of most medical schools, reflecting medicine's traditional and narrow view of the behavioral sciences as focused on techniques of managing patient behavior and the doctor-patient relationship (Bolman, 1995; Riska \& VintenJohansen, 1981). As a result, clinical health psychology has been able to achieve recognition and success in having many of its clinical practice applications (e.g., CBT techniques, assessment and interviewing skills) integrated into clerkship and residency training curricula, as well as the application of learning principles and derivative techniques to its teaching methodologies. However, topics related to domain 1 (MindBody Interactions), or the mechanisms of bio-behavioral interaction, are conspicuously less evident in medical school curricula. Hence, the IOM committee urged greater attention to defining and teaching about the mechanisms of biobehavioral interaction. Knowledge of "mind-body" or biobehavioral interaction has largely accrued through multidisciplinary research efforts from emerging new fields such as behavioral medicine, behavioral neurosciences, social neurosciences, psychoneuroendocrinology, and behavioral genetics. These are interdisciplinary fields of scientists with multidisciplinary knowledge, collaborating and integrating the principles, concepts and methodologies of their respective disciplines. Contrary to the Flexnerian legacy of competition for resources, these efforts are fostering interdisciplinary collaboration across departmental lines. 


\section{Multidisciplinary Research and Interdisciplinary Treatments}

An example of this collaborative trend is Dean Jerome Strauss' restructuring of Virginia Commonwealth University Medical Center (VCUMC), around seven multidisciplinary mission-based research efforts (cardiopulmonary disease; pathogens and the environment; maternal and child health; behavioral medicine; cancer; aging and metabolism; and regenerative medicine). Dean Strauss has established a School of Public Health, including a new multidisciplinary department of Behavioral Health, and is recruiting interdisciplinary faculty, to carry out the mission based research objectives. Central to this initiative is a focus on multi-disciplinary mission-based research teams, not discipline specific departments. In addition VCUMC is investing over $\$ 13$ million in support of 80 additional $\mathrm{Ph} . \mathrm{D}$. students in the basic health sciences, including behavioral health (www.vcuhealth.org). Dean Strauss recognizes that administrative and structural changes must accompany research and curricular change in order to truly integrate the behavioral sciences into academic medicine.

\section{Translational Medicine and Bio-Behavioral Research}

Meanwhile, translational medicine has emerged as an important "new" concept, driven by the realization that basic research should more vigorously be called upon to generate innovative clinical applications. For many medical administrators translational medicine has meant applying biological research to clinical care in the form of new pharmacological treatments, new biological measurements, new surgical procedures, and new entrepreneurial opportunities (Mancoff, Brander, Ferrone, \& Marincola, 2004). Such narrow interpretations reflect the discipline specific bias that shape and drive budgets and curriculum policies in medical education, and the discipline specific fundamentalism that impedes true integration of biomedical and behavioral research. However, Dr. Philip Pizzo, Dean of Stanford University Medical School, points out that definitions of translational medicine also include epidemiological, health outcomes and behavioral research applied to bedside or ambulatory care (Pizzo, 2002).

In October 2006 the Office of Portfolio Analysis and Strategic Initiatives of the NIH launched the Clinical and Translational Science Awards (CTSA) Consortium, beginning with 12 academic health centers across the nation. Fifty-two additional awardees will receive planning grants to prepare to join the consortium. When fully implemented in 2012, about 60 institutions will be linked to promote clinical and translational science. The purpose of the CTSA program is to assist institutions in the establishment of a "transformative, novel, and integrative academic home..." that will provide resources to...

(1) "...captivate, advance, and nurture a cadre of welltrained multi- and inter-disciplinary investigators"; (2) "...create an incubator for innovative research tools and information technologies"; and (3) "...synergize multidisciplinary and interdisciplinary ... research ... to catalyze the application of new knowledge and techniques to clinical practice..." (National Institutes of Health [NIH], 2007).

Translational medicine is already influencing the future course of medical research, medical practice and medical education. Because of its wide ranging relevance to health care, translational medicine will have a broad appeal to administrators of academic medical centers and enjoy considerable funding support from public and private agencies. The National Institute of Mental Health has made translational research for alleviating mental illness a major funding priority (Tashiro \& Mortensen, 2006). A number of the National Institutes of Health have joined in announcing a program "...to expand basic and translational research on the processes and mechanisms involved in the experience and expression of emotion..." and its contributory role in various diseases and disorders. The program announcement emphasizes interdisciplinary research strategies and the study of bio-behavioral interaction, hallmarks of translational research as well as the IOM recommendations, and should be especially interesting to clinical health psychologists (NIH, 2003).

Psychologists and other behavioral scientists working in those academic health centers that will become part of the CTSA must work diligently to insure that they are involved in the planning, development and implementation of translational research and clinical care programs, and that these programs include components and faculty from the behavioral sciences, behavioral medicine, and clinical health psychology. However, participation in interdisciplinary treatment programs targeting disorders and diseases such as cancer, for example, will require not only expertise in the role of behavioral factors in cancer onset and treatment, but also some fundamental knowledge of oncology, various organ system functions and especially immune system functioning. Similarly, competing for behavioral science career and program development awards as well as translational medicine initiatives will require the development of interdisciplinary collaborative relationships with scientists in other disciplines, and increasing multidisciplinary knowledge on the part of health psychologists.

\section{Clinical Health Psychology-A Bio-behavioral Science}

Just as these innovative concepts of medical science and practice call for greater multidisciplinary knowledge and 
interdisciplinary collaboration by physicians, so do they require a reconsideration of what is to constitute the scientific domain of clinical health psychology. The trend toward greater interdisciplinary collaboration in research argues for the increased integration of knowledge from the neurosciences and other relevant biological sciences into clinical health psychology (Calhoun \& Craighead, 2006). Doctoral training in clinical psychology purports to require knowledge of the "biological aspects" of behavior, yet the depth and breadth of that training is embarrassingly inadequate for a science that purports to be a health care profession. To insist that psychologists should only achieve mastery over a narrowly prescribed domain of scientific knowledge, and that the science of psychology should be limited only to the study of psychological phenomena and not to the biological processes that make that phenomena possible smacks of dualism and a provincial professionalism that has no place in science. Such attitudes are clearly inconsistent with the current explosion of research by psychologists into bio-behavioral mechanisms, research that demonstrates a broadening interest of psychologists in other sciences that contribute to our understanding of psychological processes. Examples include research such as efforts by psychologists to seek out the complex biobehavioral interactions and mechanisms contributing to our understanding of psychopathology (e.g., Cacioppo et al., 2007; Kreuger \& Markon, 2006), psychotherapy efficacy (e.g., Felmingham et al., 2007) fear reduction (e.g. SotresBayon, Bush, \& LeDoux, 2004), relationships between chronic stress and psychiatric and medical disorders (e.g. Miller, Chen \& Zhou, 2007), cognitive processes such as language, memory and decision-making (e.g., Fogassi \& Ferrari, 2007; Rubin, 2006; Sanfey, 2007), genetic-environmental interaction effects on behavior and psychopathology (e.g., Moffitt, Avshalom, \& Rutter, 2006), and the interaction of biological, behavioral, experiential, and socio-cultural effects upon developmental processes (e.g., Hayman, 2007; Park \& Gutchess, 2006). Clearly, psychologists' participation in multidisciplinary research and health care projects such as these requires much broader, multidisciplinary training than is currently afforded by our clinical training programs.

Clinical health psychology, of all the health care professions, has the potential to utilize its science to discover, describe, interpret, teach and clinically apply knowledge of the mechanisms of interaction between biological functions and behavioral, learning, cognitive, socio-cultural and environmental processes. With appropriate training, clinical health psychologists can be the bio-behavioral scientists that: define and explain how stress, both internal and external, impacts the neuroendocrine system and subsequently all organ systems; the mechanisms by which stress adaptation can lead to health, but also disorder, disease and illness; and how the stress response promotes learning, fosters cognition and problem solving and influences communication, behavior, socio-cultural processes, and the patient's response to disease and treatment efforts. Clinical health psychologists are also in the unique position to draw upon this knowledge to develop empirically based biobehavioral intervention strategies and programs, and to teach about bio-behavioral interaction.

In striving to become a health care profession, some psychologists, not unlike their physician colleagues, have forgotten that psychology is a science, that it is more than just a collection of insights into the doctor-patient relationship; more than just a set of interviewing techniques, assessment instruments, or treatment interventions. The object of any science is to expand its range of knowledge and establish its links to other sciences. The universe does not operate in discipline specific spheres. Significant advances in science often involve the discovery of common principles, processes and mechanisms universal to multiple sciences. If adopting a bio-psycho-social model undermines our unique understanding of the psychological component of behavior, then three decades of efforts by psychologists at trying to persuade physicians of the importance of that model would seem hypocritical. Obviously, no reasonable medical educator would so conclude. Nor should any reasonable psychologist conclude that increasing one's knowledge of biological and other sciences basic to understanding behavior, in some way dilutes the specialized expertise of psychologists. I know of no evidence that suggests that psychologists are uniquely limited or impaired in their capacity to process such new information, or that such new information will in some way deprive them of their ability to apply already familiar psychological knowledge.

The push for interdisciplinary research, prompted by translational medicine initiatives and behavioral science career development and demonstration projects, is fueling increased attention to the bio-behavioral research and teaching that the IOM Committee found so wanting in medical education. Interdisciplinary research and multidisciplinary collaboration is also fueling the development in health care of new and innovative clinical applications of these new discoveries. Such collaboration inevitably involves the sharing of knowledge, concepts, principles and methodologies. As Sonnanburg (2007) acknowledges, multidisciplinary education "equips us to benefit clients" yet the discipline of psychology seems hesitant to take the steps required to broaden the knowledge base of trainees. Clinical psychology training programs profess to include familiarity with the biological aspects of behavior in their requirements for the doctoral degree, yet preparation in this domain is woefully deficient when compared to training in the other health care professions and, despite some nominal improvements, has changed little in the past 50 years! 
As has happened in the past, other health care professions, and now other behavioral sciences, will take advantage of the opportunities and initiatives emerging in health care and academic medicine. As psychologists we can continue to set ourselves apart, or we can seek to expand our range of knowledge, not only in pharmacology, but also in the behavioral neurosciences, social neurosciences, psychoneuroendocrinology, behavioral genetics, behavioral medicine, etc. We can broaden our interdisciplinary collaborative relationships, our multidisciplinary knowledge, and our applied clinical skills, or we can watch psychology as a discipline become increasingly irrelevant to evolving medical research, medical education and health care practice.

Open Access This article is distributed under the terms of the Creative Commons Attribution Noncommercial License which permits any noncommercial use, distribution, and reproduction in any medium, provided the original author(s) and source are credited.

\section{References}

Bolman, W. M. (1995). The place of behavioral science in medical education and practice. Academic Medicine, 70, 873-878.

Cacioppo, J. T., Amaral, D. G., Blanchard, J. J., Camoron, J. L., Carter, C. S., Crews, D., et al. (2007). Social neuroscience: Progress and implications for mental health. Perspectives on Psychological Science, 2, 99-123.

Calhoun, K. S., \& Craighead, W. E. (2006). Clinical psychology in academic departments. Clinical Psychology: Science and Practice, 13, 278-281.

Carr, J. E., Emory, E. K., Errichetti, A., Bennett Johnson, S., \& Reyes, E. (2007). Integrating behavioral and social sciences in the medical school curriculum: Opportunities and challenges for psychology. Journal of Clinical Psychology in Medical Settings, 14, 33-39.

Cuff, P. A., \& Vanselow, N. A. (2004). Improving medical education: Enhancing the behavioral and social science content of medical school curricula. Washington, DC: National Academic Press.

Felmingham, K., Kemp, A., Williams, L., Das, P., Hughes, G., Peduto, A., et al. (2007). Changes in anterior cingulate and amygdala after cognitive behavior therapy of posttraumatic stress disorder. Psychological Science, 18, 127-129.

Flexner, A. (1910). Medical education in the United States and Canada. Boston: Merrymont Press.

Fogassi, L., \& Ferrari, P. F. (2007). Mirror neurons and the evolution of embodied language. Current Directions in Psychological Science, 16, 136-141.
Hayman, L. L. (2007) Behavioral medicine across the life course: Challenges and opportunities for interdisciplinary science. Annals of Behavioral Medicine, 33, 236-241.

Kreuger, R. F., \& Markon, K. E. (2006). Understanding psychopathology: Melding behavior genetics, personality, and quantitative psychology to develop an empirically based model. Current Directions in Psychological Science, 15, 113-117.

Mancoff, S. P., Brander, C., Ferrone, S., \& Marincola, F. (2004). Lost in translation: Obstacles to translational medicine. Journal of Translational Medicine, 2, 14-19.

Miller, G. E., Chen, E., \& Zhou, E. S. (2007). If it goes up, must it come down? Chronic stress and the hypothalamic-pituitraryadrenocortical axis in humans. Psychological Bulletin, 133, 25-45.

Moffitt, T. E., Avshalom, C., \& Rutter, M. (2006). Measured geneenvironment interactions in psychopathology: Concepts, research strategies, and implications for research, intervention, and public understanding of genetics. Perspectives on Psychological Science, 1, 5-27.

National Institutes of Health. (2003). Basic and translational research in emotion. Retrieved March 18, 2007, from http://grants.nih.gov /grants/guide/pa-files/PA-03-169.html.

National Institutes of Health, Office of Portfolio Analysis and Strategic Initiatives. (2007). Re-engineering the clinical research enterprise: NIH Roadmap for medical research. Retrieved March 18, 2007, from http://nihroadmap.nih.gov/clinicalresearch /overview-translational.asp.

Park, D., \& Gutchess, A. (2006). The cognitive neuroscience of aging and culture. Current Directions in Psychological Science, 15, $105-108$.

Pizzo, P. (2002). Letter from the Dean. Stanford Medicine Magazine. Retrieved March 19, 2007, from http://mednews.stanford.edu/ stanmed/2002fall/letter.html.

Riska, E., \& Vinten-Johansen, P. (1981). The involvement of the behavioral sciences in American medicine: A historical perspective. International Journal of Health Services, 11, 583-596.

Rubin, D. C. (2006). The basic-systems model of episodic memory. Perspectives on Psychological Science, 1, 277-311.

Sanfey, A. G. (2007). Decision neuroscience: New directions in studies of judgment and decision making. Current Directions in Psychological Science, 16, 151-155.

Sonnanburg, K. (2007). Pharmacotherapy and psychology. Washington Psychologist, 62, 11.

Sotres-Bayon, F., Bush, D. A. E., \& Le Doux, J. E. (2004). Emotional perseveration: An update on prefrontal-amygdala interactions in fear extinction. Learning and Memory, 11, 525-535.

Tashiro, T., \& Mortensen, L. (2006). Translational research: How social psychology can improve psychotherapy. American Psychologist, 61, 959-966. 